\title{
Prophylactic mesh reinforcement of stomas: a cost-effectiveness meta-analysis of randomised controlled trials
}

\author{
J. M. Findlay ${ }^{1,2} \cdot$ C. P. J. Wood ${ }^{1} \cdot$ C. Cunningham ${ }^{1}$
}

Received: 28 June 2017 / Accepted: 28 February 2018 / Published online: 7 May 2018

(c) The Author(s) 2018

\begin{abstract}
Background Previous meta-analyses of randomised controlled trials (RCTs) have suggested a reduction in parastomal hernias (PSH) with prophylactic mesh. However, concerns persist regarding variably supportive evidence and cost. We performed an updated systematic review and meta-analysis to inform a novel cost-effectiveness analysis.

Methods The PubMed, EMBASE and Cochrane Centre Register of Controlled Trials databases were searched (February 2018). We included RCTs assessing mesh reinforcement during stoma formation. We assessed PSH rates, subsequent repair, complications and operative time. Odds ratios (OR) and numbers needed to treat (NNT) were generated on intention to treat (ITT) and per protocol (PP) bases. These then informed cost analysis using 2017 UK/USA reimbursement rates and stoma care costs.

Results Eleven RCTs were included. Four hundred fifty-three patients were randomised to mesh (PP 412), with 454 controls (PP 413). Six studies used synthetic meshes, three composite and two biological (91.7\% colostomies; 3.64\% ileostomies, $4.63 \%$ not specified). Reductions were seen in the number of hernias detected clinically and on computed tomography scan. For the former, ITT OR was 0.23 (95\% confidence interval $0.11-0.51 ; p=0.0003 ; n=11)$; NNT $4.17(2.56-10.0)$, with fewer subsequent repairs: OR $0.29(0.13-0.64 ; p=0.002 ; n=7$; NNT16.7 (10.0-33.3). Reductions persisted for synthetic and composite meshes. Operative time was similar, with zero incidence of mesh infection/fistulation, and fewer peristomal complications. Synthetic mesh demonstrated a favourable cost profile, with composite approximately cost neutral, and biological incurring net costs.

Conclusions Reinforcing elective stomas with mesh (primarily synthetic) reduces subsequent PSH rates, complications, repairs and saves money. We recommend that future RCTs compare mesh subtypes, techniques, and applicability to emergency stomas.
\end{abstract}

Keywords Parastomal hernia $\cdot$ Surgical mesh $\cdot$ Stoma $\cdot$ Meta-analysis

\section{Introduction}

Parastomal hernias (PSH) remain common, occurring in 6\% of patients with loop ileostomies and almost half with end colostomies, and can profoundly affect quality of life, impair productivity and generate substantial healthcare costs [1].

Mesh PSH repair was originally described in 1997 [1] and is generally accepted as the most effective method. However,

J. M. Findlay

john.findlay@oncology.ox.ac.uk

1 Oxford Colorectal Centre, Churchill Hospital, Oxford University Hospitals NHS Foundation Trust, Oxford, UK

2 Department of Oncology, University of Oxford, Old Road Campus Research Building, Oxford OX3 7DQ, UK a number of recent randomised controlled trials (RCTs) have variably suggested that reinforcing stomas prophylactically with mesh reduces the risk of PSH. Consequent systematic reviews and meta-analyses have supported this position [2], and also variably suggested risk of complications to be minimal, addressing one of two major reservations towards widespread acceptance and change in practice, beyond heterogeneity of the evidence base. However, none have addressed the second: financial cost. We aimed to perform an updated systematic review and meta-analysis, informing a cost-effectiveness analysis. 


\section{Materials and methods}

\section{Literature search}

We searched the PubMed, EMBASE and Cochrane Centre Register of Controlled Trials databases up to 9 February 2018, in accordance with the Preferred Reporting Items for Systematic Reviews and Meta-Analyses and Meta-analysis Of Observational Studies in Epidemiology guidelines using the following search term: (mesh OR hernia) AND (stoma OR parastomal OR colostomy OR ileostomy) AND (randomised OR controlled OR trial). Bibliographies of retrieved articles were searched. Two authors (JMF and CPJW) screened articles independently.

\section{Inclusion and exclusion criteria}

We included English language RCTs randomising patients undergoing stoma formation (loop/end ileostomy/colostomy) to mesh reinforcement (synthetic/composite/biological) or not.

\section{Endpoints}

Primary outcomes were development of PSH (clinically and/ or radiologically detected, as defined in individual studies) and subsequent repair. These were then used to inform a cost-effectiveness analysis. Our secondary outcomes were complications (as defined by individual studies) and operating time [3].

\section{Data collection}

Data were extracted independently (JMF and CPJW). We contacted one study's authors to clarify ITT and PP results [4].

\section{Assessment of bias and evidence quality}

Bias was assessed using the Cochrane Collaboration 'Risk of bias' tool [5], with recommendations stratified using GRADE [6].

\section{Meta-analysis and other statistical analysis}

Meta-analysis was performed using RevMan v5.2 (The Cochrane Collaboration); additional analyses using R v3.02 (R Core Team). Heterogeneity was quantified by $I^{2}$ and Chisquare. Odds ratios (OR), absolute risk reduction (ARR) and 95\% confidence intervals (CI) were calculated used random effects when $I^{2}>50 \%$. Both intention to treat (ITT; patients randomised to treatment arms, irrespective of subsequent protocol violations), and per protocol (PP; only patients receiving treatment and follow-up as stipulated) analyses were performed. Funnel plots were inspected for asymmetry. Perceived publication bias was corrected using the 'trim and fill' method [7]. $p<0.05$ was considered significant. Sensitivity analyses were conducted.

\section{Cost-effectiveness analysis}

We performed a two-tiered analysis. The first (most conservative) comprised the cost of elective PSH repair and mesh. Net inpatient PSH repair costs were obtained from 2017 United States of America (USA) Medicare national average reimbursement rates, and United Kingdom (UK) National Health Service tariff, (USD\$7572/\$9989/\$17,143; and GBP£1775/£2646/£4328 for no, intermediate and major comorbidities, respectively; procedure codes MSDRG Codes 353-355). Mesh costs were obtained from Medtronic, Ethicon and Elemental Healthcare for representative $10 \times 15 \mathrm{~cm}$ synthetic, composite and biological meshes. Mean costs were: $£ 32.77 / \$ 42.60, £ 296.64 / \$ 385.64$ and $£ 1650 / \$ 2145$, respectively. A cost range was calculated based on cheapest and most expensive meshes and tariffs.

The second tier included additive stoma care for patients with a PSH per year for 2 years (typical follow-up of included RCTs). This was estimated by assuming PSH resulted in $25 \%$ more frequent leakage with a commensurate increase in cost of appliances and care (£3024.80-4315/\$3932.5-5609.50) $[8,9,10]$.

\section{Results}

\section{Literature search}

One thousand one hundred twenty-three studies were identified; 32 full texts were appraised; 11 RCTs were included (Fig. 1). Four hundred fifty-three patients were randomised to mesh (per protocol (PP) 412), with 454 controls (PP 413). Six studies used synthetic mesh [3, 4, 11-14], three composite [15-17] and two biological [18, 19]. Eight studies only included end colostomy, one only loop ileostomy [19], either ileostomy or colostomy [18], and 1 unspecified stoma formation [14] Overall, 33 patients (PP; 3.64\%) had ileostomy formation, and $832(91.7 \%)$ colostomy [42 (4.63\%) not specified]. One hundred eighty-three patients (21.6\%) underwent a laparoscopic operation, $523(61.8 \%)$ open [140 (16.5\%) not specified]. Five studies included only abdominoperineal excision for rectal/pelvic cancer [12, 13, 15-17]; one end ileostomy/colostomy for low rectal cancer [14]; three laparotomy with end colostomy $[3,4,11]$; one permanent end ileostomy/colostomy for any indication [18]; and 
PRIS MAE PRISMA 2009 Flow Diagram

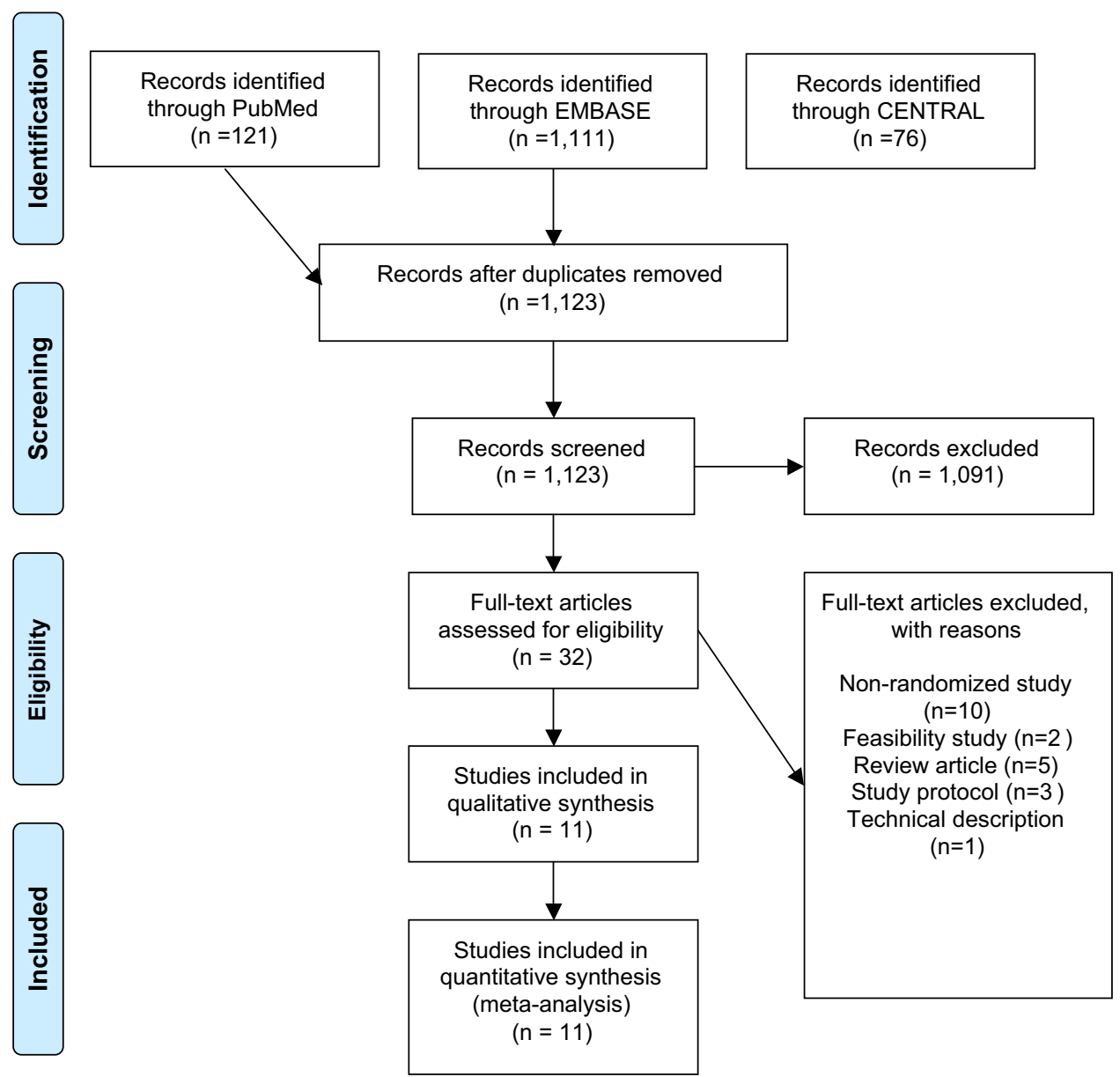

Fig. 1 Preferred reporting items for systematic reviews and meta-analyses (PRISMA) diagram

one loop ileostomies after major anorectal surgery [19]. All studies included elective patients, other than two (including four and one emergency laparotomies, respectively) [3, 11]. Two studies excluded patients with a body mass index $>35$ $[13,18]$. Follow-up varied: up to 12 months in 7 [3, 4, 13, 15-17, 19], 24 months in 2 [14, 18]; 1-4 years [12] and $1-5$ years $[11]$.

\section{Endpoints}

In patients randomised to prophylactic mesh, significantly fewer PSH were detected clinically: intention to treat (ITT) OR $0.23(0.11-0.51 ; p=0.0003 ; n=11$; Table 1; GRADE recommendation moderate; Table 2). Number need to treat (NNT) was 5.00 (3.22-10.0). PP OR was 0.37 (0.26-0.53). Lesser reductions were seen for CT-detected hernias: OR $0.43(0.26-0.71 ; p=0.001 ; n=9)$. Subgroup analysis demonstrated significance for synthetic and composite meshes, and end colostomies. PP results were similar.

This translated into fewer subsequent repairs: OR 0.29 (0.13-0.64; $p=0.002)$; NNT 16.7 (10.0-33.3); GRADE recommendation low, persisting for synthetic mesh: NNT 11.1 (7.14-25.0). There was zero incidence of mesh infection/fistulation. There were fewer minor peristomal complications with mesh (GRADE recommendation very low): ITT OR $0.48(0.30-0.77), p=0.002 ; n=9$. Operative time was similar: mean difference 5.16 min (-13.4-23.8; $p=0.590 ; n=6)$.

\section{Evidence quality}

Overall, quality ranged from very low to moderate (Table 2). The evidence for PSH reductions was moderate (further research being unlikely to change the effect 
Table 1 Effects on clinically detected hernias and subsequent repair

\begin{tabular}{|c|c|c|c|c|c|c|c|}
\hline Analysis & OR & $95 \% \mathrm{CI}$ & $p$ & $n$ studies & $I^{2 *}(\%)$ & Effects model & $\begin{array}{l}\text { Adjusted for } \\
\text { publication } \\
\text { bias? }\end{array}$ \\
\hline \multicolumn{8}{|c|}{ Clinically detected hernias } \\
\hline \multicolumn{8}{|c|}{ All meshes } \\
\hline ITT & 0.23 & $0.11-0.51$ & 0.0003 & 9 & 66 & Random & Yes \\
\hline PP & 0.37 & $0.26-0.53$ & 0.0008 & 9 & 67 & Random & Yes \\
\hline \multicolumn{8}{|c|}{ Synthetic mesh } \\
\hline ITT & 0.11 & $0.05-0.22$ & $<0.0001$ & 6 & 81 & Random & No \\
\hline PP & 0.19 & $0.05-0.71$ & 0.001 & 6 & 81 & Random & No \\
\hline \multicolumn{8}{|c|}{ Biological mesh } \\
\hline ITT & 0.59 & $0.21-1.64$ & 0.310 & 2 & 37 & Fixed & No \\
\hline PP & 0.41 & $0.14-1.27$ & 0.130 & 2 & 6 & Fixed & No \\
\hline \multicolumn{8}{|c|}{ End colostomy } \\
\hline ITT & 0.20 & $0.07-0.58$ & 0.0001 & 6 & 80 & Random & No \\
\hline PP & 0.21 & $0.07-0.69$ & $<0.0001$ & 6 & 81 & Random & No \\
\hline \multicolumn{8}{|c|}{ CT-detected hernias } \\
\hline ITT & 0.43 & $0.26-0.71$ & 0.0010 & 8 & 49 & Random & Yes \\
\hline PP & 0.40 & $0.20-0.77$ & 0.0060 & 8 & 62 & Random & Yes \\
\hline \multicolumn{8}{|c|}{ Parastomal hernia repair } \\
\hline \multicolumn{8}{|c|}{ All meshes } \\
\hline ITT & 0.37 & $0.24-0.55$ & $<0.0001$ & 7 & 32 & Fixed & Yes \\
\hline PP & 0.33 & $0.16-0.68$ & 0.003 & 7 & 62 & Random & Yes \\
\hline \multicolumn{8}{|l|}{ Synthetic } \\
\hline ITT & 0.16 & $0.04-0.55$ & $<0.0001$ & 5 & 81 & Random & No \\
\hline PP & 0.15 & $0.04-0.57$ & 0.006 & 4 & 0 & Fixed & No \\
\hline \multicolumn{8}{|c|}{ Composite } \\
\hline ITT & 0.55 & $0.13-2.36$ & 0.420 & 3 & 0 & Fixed & No \\
\hline PP & 0.57 & $0.13-2.42$ & 0.440 & 3 & 0 & Random & No \\
\hline \multicolumn{8}{|c|}{ Biological } \\
\hline ITT & 0.47 & $0.11-1.99$ & 0.310 & 1 & NA & Fixed & No \\
\hline PP & 0.46 & $0.09-2.44$ & 0.360 & 1 & NA & Fixed & No \\
\hline \multicolumn{8}{|c|}{ End colostomy } \\
\hline ITT & 0.27 & $0.10-0.75$ & 0.0100 & 6 & 0 & Fixed & No \\
\hline PP & 0.28 & $0.10-0.80$ & 0.020 & 6 & 0 & Fixed & No \\
\hline
\end{tabular}

$O R$ odds ratio, $C I$ confidence interval, $C T$ computed tomography, ITT intention to treat, $P P$ per protocol

*Before adjusting for publication bias direction, but likely to change the estimate). This was affected by serious risk of bias and probable publication bias, but enhanced by consistency of effect. Evidence for biological meshes was very low, indicating high uncertainty (very serious bias, indirectness including use of mesh for temporary loop ileostomies, and imprecision in effect sizes). Evidence for the reductions in repairs were low, limited mainly by very serious potential bias (inevitable variability in thresholds for repair), indicating that further research may affect direction of effect. Quality for complication reductions was very low (very serious risk of bias, particularly in complication reporting and definitions, and imprecision in effect sizes).

\section{Cost analysis}

Routine use of synthetic mesh would save money (Table 3). Considering only operative costs, in the USA overall cost per patient was USD\$ -622.36 to -1513.21 (GBP£ +135.91 to -365.91$)$ Including stoma costs, this was reduced further. Composite meshes were roughly cost neutral when considering operative costs, with lesser savings when considering stoma costs. The expense of biological mesh resulted in net costs. 
Table 2 GRADE recommendations

\begin{tabular}{|c|c|c|c|c|c|c|c|}
\hline Analysis & LOE & Risk of bias & Inconsistency & Indirectness & Imprecision & Publication bias & Quality \\
\hline \multicolumn{8}{|c|}{ Clinically detected hernias } \\
\hline All & $\mathrm{RCT}(+4)$ & Serious $(-1)$ & Consistent $(+1)$ & None & None & Detected $(-1)$ & Moderate \\
\hline Synthetic & $\mathrm{RCT}(+4)$ & Serious $(-1)$ & None & None & None & Not detected & Moderate \\
\hline Biological & $\mathrm{RCT}(+4)$ & Very serious $(-2)$ & None & Serious $(-1)$ & Serious $(-1)$ & Not detected & Very low \\
\hline \multicolumn{8}{|c|}{ CT-detected hernias } \\
\hline All & $\mathrm{RCT}(+4)$ & Serious $(-1)$ & Consistent $(+1)$ & None & None & Detected $(-1)$ & Moderate \\
\hline Synthetic & $\mathrm{RCT}(+4)$ & Serious $(-1)$ & None & None & None & Not detected & Moderate \\
\hline Composite & $\mathrm{RCT}(+4)$ & Serious $(-1)$ & None & None & None & Not detected & Moderate \\
\hline \multicolumn{8}{|c|}{ Complications } \\
\hline All & $\mathrm{RCT}(+4)$ & Very serious $(-2)$ & None & None & Serious $(-1)$ & Detected $(-1)$ & Very low \\
\hline Synthetic & $\mathrm{RCT}(+4)$ & Very serious $(-2)$ & None & None & Serious $(-1)$ & Not detected & Very low \\
\hline Composite & $\mathrm{RCT}(+4)$ & Very serious $(-2)$ & None & None & Serious $(-1)$ & Not detected & Very low \\
\hline \multicolumn{8}{|c|}{ Hernia repair } \\
\hline All & $\mathrm{RCT}$ & Very serious $(-2)$ & None & None & None & Not detected & Low \\
\hline \multicolumn{8}{|c|}{ Operative time } \\
\hline ITT & $\mathrm{RCT}$ & Very serious $(-2)$ & None & Serious $(-1)$ & Serious $(-1)$ & Not detected & Low \\
\hline
\end{tabular}

$R C T$ randomised controlled trial, $L O E$ level of evidence, $C T$ computed tomography

Table 3 Cost-effectiveness

\begin{tabular}{lcll}
\hline Mesh & NNT & \multicolumn{2}{l}{ Net cost per patient } \\
\cline { 3 - 4 } & & Lowest & Highest \\
\hline USD \$ & & & \\
Synthetic & 11.1 & -1513.21 & -622.36 \\
Composite & 33.3 & $-278.80-$ & +468.00 \\
Biological & 20 & +792.85 & +2351.40 \\
GBP £ & & & \\
Synthetic & 11.1 & $-365.91--$ & $+135.91-$ \\
Composite & 33.3 & $+106.03--$ & +306.70 \\
Biological & 20 & $+983.6-$ & +2011.25 \\
Plus additive stoma costs & & \\
USD \$ & & & \\
Synthetic & 3.45 & -2138.58 & -1192.29 \\
Composite & 6.68 & -698.68 & +173.65 \\
Biological & 16.67 & +624.60 & +2233.45 \\
GBP £ & & & -552.32 \\
Synthetic & 3.45 & -991.27 & +80.27 \\
Composite & 6.68 & -216.95 & +1920.52 \\
Biological & 16.67 & +854.18 & \\
\hline
\end{tabular}

$N N T$ number needed to treat, USD US dollar, GBP British pound sterling

\section{Discussion}

In the most up-to-date meta-analysis of 11 RCTs involving 907 patients, we found reinforcing elective stomas with prophylactic mesh reduced the incidence of subsequent
PSH and repair. There was no significant increase in operative time, with substantial cost savings for synthetic meshes, and fewer peristomal complications. The evidence was less clear (and of lower quality) for composite and biological meshes, with the extra cost of the latter seeming to subsume potential savings.

These findings agree with recent meta-analyses [2]. However, none have assessed costs, anecdotally perceived to be significant and hence a barrier to changes in practice. As such we believe our findings are novel, and provide context to the evolving evidence base. This notwithstanding, we acknowledge a number of limitations, beyond those of constituent study heterogeneity, bias and methodology. We used GRADE recommendations to provide context, but we were unable to perform meaningful meta-regression on the basis of individual study quality. Similarly, we were unable to compare stoma types, other than analysis of end colostomy.

Ours is also the first meta-analysis to include the recent publication by Odensten et al. [3]. This was notable both as the largest study to date, with relatively little bias and its finding of a lack of effect. The reasons for this are unclear; however, the rates of PSH in both arms of the trial were high, at approximately $30 \%$.

Our cost analysis was by necessity constrained by the very limited data available regarding cost to patients, and projections by length of study follow-up. It is very likely that our estimates are conservative: NNT would likely reduce further with time, and we could not reliably account for significant additional costs, including the economic costs to patients who develop hernias (for example time off work) beyond stoma care. Furthermore, it is likely that commensurate 
benefits in quality of life would be seen beyond the financial, but these areas have received little attention in the literature.

We recommend that these be explored in the next generation of RCTs and furthermore, that these compare mesh types and reinforcement approach. However, perhaps most pressing is whether reinforcement is of benefit in patients undergoing emergency stomas, particularly a Hartmann's procedure. A substantial proportion of these patients never undergo reversal, whilst they are perhaps most vulnerable to the consequences of PSH.

\section{Conclusions}

We found that reinforcing stomas with mesh reduces subsequent PSH rates, repair and complications, and should be considered routinely. Synthetic mesh has the best evidence profile and results in potentially substantial cost savings. The additional cost of composite and biological mesh does not yet appear to confer any additional benefit.

\section{Compliance with ethical standards}

Conflict of interest The authors declare that they have no conflict of interest.

Ethical approval This article does not contain any studies with human participants or animals performed by any of the authors.

Informed consent As a meta-analysis of published data formal consent was not required.

Open Access This article is distributed under the terms of the Creative Commons Attribution 4.0 International License (http://creativeco mmons.org/licenses/by/4.0/), which permits unrestricted use, distribution, and reproduction in any medium, provided you give appropriate credit to the original author(s) and the source, provide a link to the Creative Commons license, and indicate if changes were made.

\section{References}

1. Carne PW, Robertson GM, Frizelle FA (2003) Parastomal hernia. Br J Surg 90:784-793

2. Patel SV, Zhang L, Chadi SA, Wexner SD (2017) Prophylactic mesh to prevent parastomal hernia: a meta-analysis of randomized controlled studies. Tech Coloproctol 21:5-13

3. Odensten C, Strigard K, Rutegard J et al (2017) Use of prophylactic mesh when creating a colostomy does not prevent parastomal hernia: a randomized controlled trial-STOMAMESH. Ann Surg (ePub ahead of print)
4. Brandsma HT, Hansson BM, Aufenacker TJ et al (2017) Prophylactic mesh placement during formation of an end-colostomy reduces the rate of parastomal hernia: short-term results of the Dutch PREVENT-trial. Ann Surg 265:663-669

5. Higgins JP, Green, S (2011) Cochrane handbook for systematic reviews of interventions version 5.1.0. In: The cochrane collaboration

6. Guyatt GH, Oxman AD, Vist GE et al (2008) GRADE: an emerging consensus on rating quality of evidence and strength of recommendations. BMJ 336:924-926

7. Peters JL, Sutton AJ, Jones DR et al (2007) Performance of the trim and fill method in the presence of publication bias and between-study heterogeneity. Stat Med 26:4544-4562

8. Meisner S, Lehur PA, Moran B et al (2012) Peristomal skin complications are common, expensive, and difficult to manage: a population based cost modeling study. PLoS ONE 7:e37813

9. Randall J, Lord B, Fulham J, Soin B (2012) Parastomal hernias as the predominant stoma complication after laparoscopic colorectal surgery. Surg Laparosc Endosc Percutan Tech 22:420-423

10. Tan EK, Vaizey C, Cornish J et al (2008) Surgical strategies for faecal incontinence: a decision analysis between dynamic graciloplasty, artificial bowel sphincter and end stoma. Colorectal Dis 10:577-586

11. Janes A, Cengiz Y, Israelsson LA (2009) Preventing parastomal hernia with a prosthetic mesh: a 5-year follow-up of a randomized study. World J Surg 33:118-121

12. Lambrecht JR, Larsen SG, Reiertsen O et al (2015) Prophylactic mesh at end-colostomy construction reduces parastomal hernia rate: a randomized trial. Colorectal Dis 17:O191-O197

13. Serra-Aracil X, Bombardo-Junca J, Moreno-Matias J et al (2009) Randomized, controlled, prospective trial of the use of a mesh to prevent parastomal hernia. Ann Surg 249:583-587

14. Tarcoveanu E, Vasilescu A, Cotea E et al (2014) Parastomal hernias - clinical study of therapeutic strategies. Chirurgia (Bucur) 109:179-184

15. Lopez-Cano M, Lozoya-Trujillo R, Quiroga S et al (2012) Use of a prosthetic mesh to prevent parastomal hernia during laparoscopic abdominoperineal resection: a randomized controlled trial. Hernia 16:661-667

16. Lopez-Cano M, Serra-Aracil X, Mora L et al (2016) Preventing parastomal hernia using a modified sugarbaker technique with composite mesh during laparoscopic abdominoperineal resection: a randomized controlled trial. Ann Surg

17. Vierimaa M, Klintrup K, Biancari F et al (2015) Prospective, randomized study on the use of a prosthetic mesh for prevention of parastomal hernia of permanent colostomy. Dis Colon Rectum 58:943-949

18. Fleshman JW, Beck DE, Hyman N et al (2014) A prospective, multicenter, randomized, controlled study of non-cross-linked porcine acellular dermal matrix fascial sublay for parastomal reinforcement in patients undergoing surgery for permanent abdominal wall ostomies. Dis Colon Rectum 57:623-631

19. Hammond TM, Huang A, Prosser K et al (2008) Parastomal hernia prevention using a novel collagen implant: a randomised controlled phase 1 study. Hernia 12:475-481 